\title{
DeepRS: Deep-learning Based Network-Adaptive FEC for Real-Time Video Communications
}

\author{
Sheng Cheng, Han Hu, Xinggong Zhang*, Zongming Guo \\ Wangxuan Institute of Computer Technology \\ Peking University \\ Email: \{chaser_wind, huhan951753, zhangxg, guozongming\}@pku.edu.cn
}

\begin{abstract}
As real-time multimedia streaming thriving, Forward Error Correction (FEC) methods have been studied and applied extensively these years. Most of researchers paid their attention to the coding algorithms, attempted to balance the trade off between recovery ratio and delay with fewer redundance. However, when packet loss pattern changes dynamically, the redundance waste is too serious to be ignored. In this work, we propose a novel algorithm which adjusts the redundance ratio of FEC encoder according to the prediction of packet loss. Receivers are additionally required to feedback observed packet loss pattern. Streaming sender collects the feedbacked packet loss pattern and predicts the number of packet loss in the incoming short period. As for implementation, we adopt long short-term memory (LSTM) network as our deep learning algorithm, and exquisitely embed it in our adaptive FEC system. With the extensive experiments, our proposed scheme outperforms other FEC methods greatly both in the simulations and evaluations on traces observed from the real world.
\end{abstract}

Index Terms-Deep-learning, Network-adaptive streaming, Forward error correction (FEC)

\section{INTRODUCTION}

Real-time video streaming is getting more and more prevalent in these years. Network video streaming is expected to account for $82 \%$ of Internet traffic by 2022 . In the meanwhile, a growing share of network video will take the form of live streaming video [1], and Real-time Video Communication (RTC) is drawing increasing attention of users and researchers. However, packet loss is proved to be a critical problem for RTC, because it could cause distortion and decoding error, thus degrading users' Quality of Experience (QoE). Considering the demand of RTC applications like video conferences that one way delay is limited to no more than $200 \mathrm{~ms}$ according to the standard of International Telecommunication Union (ITU), it is challenging to recover packet losses via restransmission on Internet. Automatic Repeat-reQuest (ARQ) is a traditional error correction method, but its recovery delay is longer than an RTT. In order to solve this problem efficiently, application-layer Forward Error Correction (FEC) [2] has been proposed. Application-layer FEC applies error-resilient coding algorithms to source packets to generate redundant packets, which can recover the ever lost source packets with relatively low latency when packet loss happens. It is validated that

* Dr. Xinggong Zhang is the corresponding author.

This work was supported by the National Key R\&D program of China (2019YFB1802701, 2018YFB0803702), Alibaba Innovative Research (AIR) Program (XT622018001708).

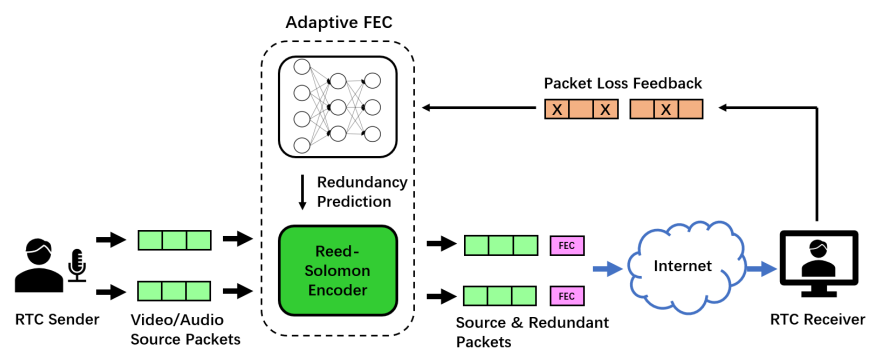

Fig. 1. The framework of deep-learning based adaptive FEC. DeepRS collects delayed feedback from receiver, makes prediction on future packet loss using LSTM model, and adjusts the parameters of RS encoder.

application-layer FEC is an effective technique to achieve lowlatency packet transmissioin, but it is still a big challenge for application-layer FEC to improve the efficiency under timevarying packet loss pattern.

There are two main categories of the existing works addressing the issue: coding scheme and adaptive FEC.

- Coding scheme focuses on devising new FEC coding algorithms to enhance the efficiency of packet loss recovery. For instance, fountain code [3], Raptor code [4], [5] and Reed-Solomon (RS) code [6], [7] have been studied. Moreover, Xiao et.al. put forward the expanding window approach to apply unequal protection to different packets of video/audio data on the basis of their importance, so as to improve QoE in RTC [8]. However, these works are based on the hypothesis that network packet loss pattern is regular or fixed, which is not practical on real Internet. It is often the case that the redundant packets of some blocks are wasted due to the absence of packet loss, or they can not help owing to the overfull packet loss.

- Adaptive FEC is first suggested to cope with the challenge of dynamic loss pattern by Padhye et.al [9]. Atiya et.al came up with a nonlinear prediction approach to realize automatic feature selection [10], and Fong et.al. combined an ingenious coding scheme along with a network adaptive algorithm for parameter update [11]. Nevertheless, all of them simply take the historical network pattern as the prediction of future pattern, ignoring the possible complicated relationship between history and future. When network conditions change frequently, these 


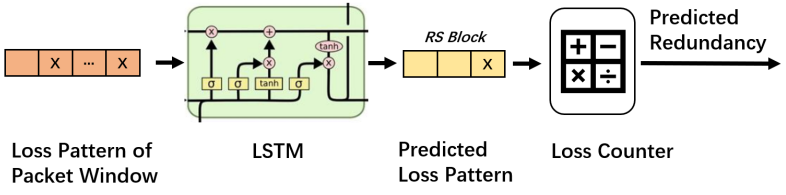

Fig. 2. The diagram of LSTM Module. LSTM takes the vector of historical pattern as input and predicts future pattern, then the Loss Counter accumulates number of lost packets in the future pattern and generate the output.

techniques will not work well as expected.

To make good use of the contextual relationship between history and future, we propose DeepRS, a novel FEC algorithm which predicts network packet loss with deep neural network, dynamically adjusts the redundancy ratio, and improves the efficiency of FEC scheme markedly. The framework of deep-learning based adaptive FEC is shown in Fig. 1. DeepRS predicts the packet loss based on the feedback from receiver, determines the amount of redundant packets and applies RS coding algorithm to encode this video block. In order to make the best of the contextual relevance of network loss pattern, we propose a prediction method of packet loss based on Long Short-Term Memory (LSTM) network. A large number of experiments on simulation and real Internet traces show that the recovery ratio of DeepRS is $70 \%$ higher than the compared algorithms in the case of a fixed total redundancy rate, and DeepRS can realize adaptive FEC redundancy in any network dynamic. To the best of our knowledge, this is the first time to verify that the deep-learning FEC is able to improve FEC efficiency significantly. It may open a new door to error correction coding research.

The main contributions of this paper are listed as follows.

1) Design an LSTM model to solve the problem of predicting dynamic network packet loss.

2) Come about the packet loss counting method to solve the problem of predicting loss pattern accurately.

3) Propose the block gap prediction method to solve the problem of delayed feedback.

The remainder of this paper is organized as follows. Section II covers concrete definition and construction of DeepRS. In Section III, we present the setup of our experiments as well as the evaluations. And eventually we come to the conclusion in Section IV

\section{SYSTEM DESIGN}

DeepRS consists of two main modules, LSTM network and RS encoder. At the every beginning of encoding procedure, after collecting information from receiver, the LSTM module predicts the incoming network packet loss, and then the RS encoder generates redundant packets according to the results of LSTM.

\section{A. DeepRS Packet Loss Prediction}

DeepRS takes advantage of LSTM model to predict network packet loss. LSTM [12] is a very popular category of deep

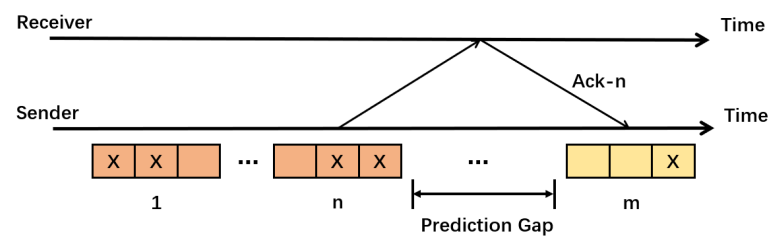

Fig. 3. Gap resides between the input vector and the output vector. This design makes DeepRS adapted for real-time video streaming.

learning model, which has been proven to have powerful capability to process sequenced data, for example, the word sequences in natural language. An LSTM cell mainly contains three gates: forget gate, input gate and output gate. These gates are responsible for eliminating useless historical information, updating the current state of cell according to the input and generating output separately. The particular structure enables LSTM to unearth the contextual relevance of sequential data more efficiently.

The contextual correlation between historical packet loss pattern and future packet loss pattern is a natural idea. Historical packet loss pattern implicitly indicates the state of the network, which plays a leading role in future packet loss. This intuition inspires us that applying LSTM model can be a wonderful method to make prediction on network packet loss. Moreover, It has been clarified that network conditions do not change drastically in a short period [13] and packets share similar states under the same network conditions [14], which indicates that network fluctuation possibly has regularity to some extent. These conclusions are firm support that encourages us to use learning based method, or rather, LSTM model, to predict the packet loss of next sending block by learning from the historical loss pattern.

The structure of LSTM module is shown in Fig. 2. LSTM takes the historical loss pattern as input and outputs the predicted packet loss pattern. In the training step, a large amount of historical packet loss sequences are collected and split into blocks containing 6 packets. Each sample of data set contains packet loss pattern of 5 blocks as input and the loss pattern of the next block as label. In the reference stage, LSTM module predicts the packet loss pattern of incoming coding block based on the feedback of 5 blocks from receiver.

\section{B. Unpredictable Loss Pattern}

General LSTM network is used to learn a mapping from a sample vector to a target vector, but the form of output is not suitable for solving the problem of packet loss. According to the network conditions, the amount of lost packets in an incoming block can be predicted by learning from the historical pattern, but the loss state of each packet is harder to be exactly determined because of randomness. What the LSTM model learns from is the historical loss pattern, which is mainly influenced by the network conditions, so that it can reflect the future packet loss to some extent. Nevertheless, the loss position does not have certain relationship with the 


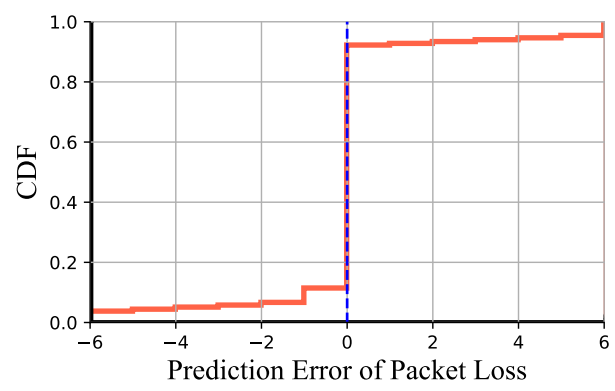

Fig. 4. Prediction error distribution of DeepRS under simulation traces indicates the majority of predictions are completely accurate.

historical loss pattern. In a word, the future loss pattern is sometimes unpredictable, which makes it improper to be the final output.

Fortunately, from the perspective of FEC, actually we do not need to know the loss state for each packet accurately. This character enables us to avoid the difficulty of directly predicting loss pattern. We use Loss Counter to predict the number of packets lost in each block based the loss pattern as the final output. Since it is directly affected by the network conditions just like the historical loss pattern, the count of packet loss can be exactly derived by the fully trained LSTM network.

As is shown in Fig. 2, we attach Loss Counter after LSTM, transforming the loss pattern into number of lost packets. According to the predicted number of lost packets, DeepRS decides how many redundant packets ought to generated in this block.

\section{Delayed Feedback}

Because of the existence of RTT, the packet loss pattern feedback from receiver is delayed. DeepRS is not able to obtain the newest network status when a video block is about to be sent. Therefore, we need to make prediction on incoming packet loss pattern according to packet loss an RTT ago.

To handle this problem, we propose the block gap method for prediction and inference, which is shown in Fig. 3. In the training step, we insert a Prediction Gap between the historical loss pattern and the blocks to be predicted. The Prediction Gap contains some blocks, whose sending time is an RTT in total, to analog the effect of network delay. In the inference step, DeepRS can still work in spite of the existence of RTT thanks to the design of Prediction Gap in the training step.

\section{EXPERIMENTS AND EVALUATIONS}

To validate the efficiency of DeepRS, we carry out tracebased evaluations, both on simulation traces and real Internet packet loss traces.

\section{A. Setup}

1) Dataset: Gilbert-Elliot (GE) channel is acknowledged as a common simulation environment of network packet delivery. In Section III-B, we carry out experiments on simulation traces

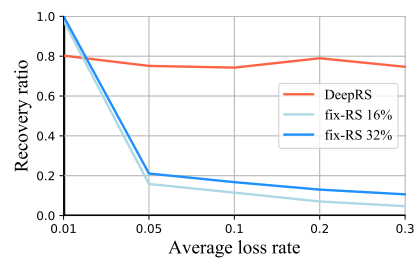

(a) Recovery ratio

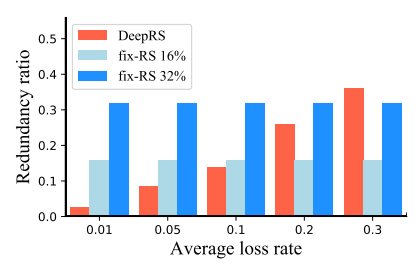

(b) Redundancy ratio
Fig. 5. Performance comparison under different loss rate shows that DeepRS keeps its performance stable by adjusting its redundancy ratio.

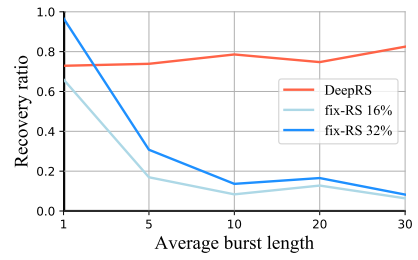

(a) Recovery ratio

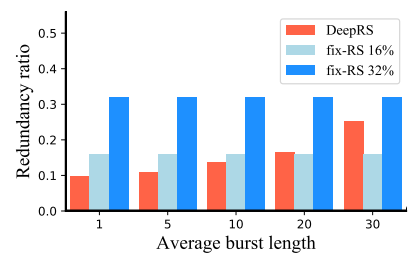

(b) Redundancy ratio
Fig. 6. Performance comparison under different burst length also shows the stable performance and the adaptive redundancy ratio of DeepRS.

generated by GE channel and evaluate the performance of DeepRS.

Real Internet packet loss traces published by Fu et.al [15] contains transmission meta data of tens of thousands of packets in 802.15 WPAN network. The performance of DeepRS on the Internet packet loss dataset is evaluated in Section III-C.

2) Underlying FEC algorithm: As the name of DeepRS indicates, RS code is selected as the underlying FEC algorithm. In our implementation, $b$ source packets are grouped as a block and the encoder generates $k$ redundant packets based on these source packets. Once $b$ or more packets, including both source and redundant packets, are collected by the receiver, the original data can be recovered by solving a matrix equation. For simplicity, we assume redundant packets will not be lost.

We choose naive RS methods as the contrast algorithms, since they are widely used in multimedia streaming. We use Fix-* RS to represent these algorithms, while the redundancy ratio is static. * indicates the fixed redundancy ratio.

3) Metrics: In performance comparison, we take the following measurement metrics into consideration:

- Recovery Ratio. The ratio of recovered packets to all lost packets. For instance, recovery ratio is 1 when all lost packets are recovered. Conversely, recovery ratio is 0 if all lost packets cannot be recovered.

- Redundancy Ratio. The ratio of redundant packets to source packets. For instance, if RS module generates $k$ FEC packets with a block including $b$ source packets, the redundancy ratio is $\frac{k}{b}$.

\section{B. Experiments on Simulation}

Simulation traces contain 10,000 samples generated from the output of GE channel. For each sample, we collect the output vector of length $7 b$, then take the front part of length 


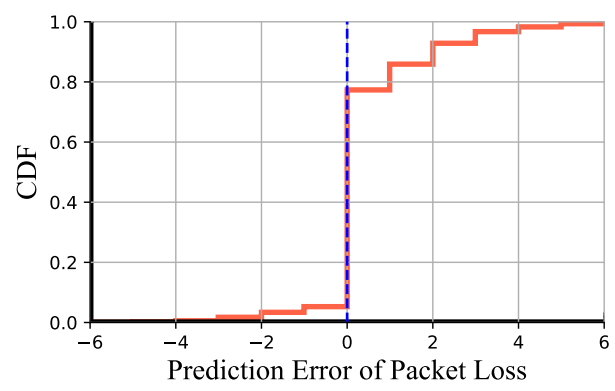

Fig. 7. Prediction error distribution under real Internet traces also shows high prediction accuracy of DeepRS.

$5 b$ as input vector, ignore the Prediction Gap whose length is $b$, and take the last part of length $b$ as label.

1) Analysis of Prediction Error Distribution: In this section, experiments are demonstrated on a GE channel given a group of fixed parameters. Simulation traces are split into training set, validation set and test set which account for $60 \%$, $20 \%, 20 \%$ of whole data set separately. Finally, results on test set are illustrated in Fig. 4 after the loss function converges on validation set.

According to the results shown in Fig. 4, DeepRS predicts the number of packet loss accurately (zero error) with $70 \% \sim 80 \%$ probability. This result validates our insight preliminarily.

2) Performance under Different Loss Rate: In this section, we evaluate the performance of DeepRS with diverse average loss rate given fixed average burst length. We choose fix-16\% RS and fix-32\% RS as contrast algorithms. Let average burst length be 10 packets, average loss rate $1 \%, 5 \%, 10 \%, 20 \%$, $30 \%$ are selected. The trend of recovery ratio and redundancy ratio are shown in Fig. 5

According to the results, DeepRS is able to alter redundancy ratio under distinct average loss rates. As a result, its recovery ratio stays relatively stable while the performance of fixed redundancy methods plummet as average loss rate grows up. In a word, DeepRS outperforms contrast algorithms greatly.

3) Performance under Different Burst Length: Similar to Section III-B2, experiments are made under several average burst lengths given a fixed average loss rate. Average loss rate is set to $10 \%$, and average burst length varies from 1 to 30 , while the same contrast algorithms are selected.

The results in Fig 6 show that the behavior of DeepRS is similar to Section III-B2. According to Fig. 6(b), it is clear that DeepRS adjusts its redundancy ratio dynamically under GE channels with different average burst lengths. Fig. 6(a) shows that DeepRS keeps high recovery ratio while the contrast algorithms work worse if average burst length is getting longer.

\section{Experiments on Real Internet Traces}

In this section, we extract packet loss information from the Internet traces. In total, we split 20192 traces into training part, validation part and test part, which account for $60 \%, 20 \%$

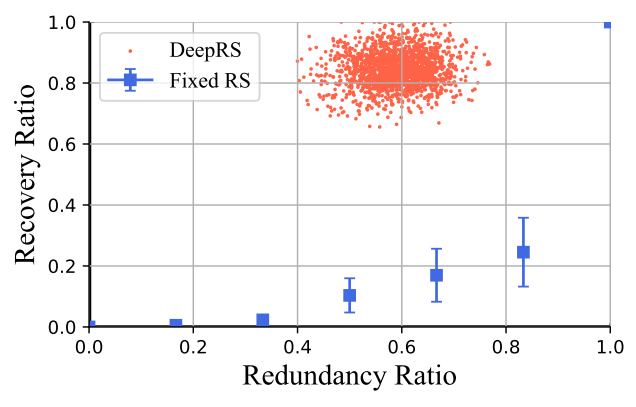

Fig. 8. Performance evaluation. DeepRS applies different redundancy ratio for each trace, so the results are shown as discrete points. For each fixed RS method, the $95 \%$ confidence interval is plotted.

and $20 \%$ of original data separately. Next, we apply sliding window approach to generate samples from each part of data. The same standard machine learning process is carried out as mentioned in Section 4 Prediction error distribution is shown in Fig. 7, which indicates that DeepRS is prone to predict number of packet loss accurately with approximately $70 \%$ probability. This figure implies that DeepRS also works well in real Internet environment.

What's more, the trade off between recovery ratio and redundancy ratio is explored and the performance of DeepRS and fix-RS are shown in Fig 8 In this experiment, 7 distinct fix-RS methods are selected as contrast algorithms. As is revealed in Fig. 8. DeepRS significantly outperforms traditional RS code methods with fixed redundancy. DeepRS recovers approximately $80 \%$ packets while fix-RS methods help little on packet recovery. Notice that the recovery ratio of DeepRS is multiple of fix-RS method when redundancy ratio is equal, which means that DeepRS is able to achieve higher recovery ratio with much less extra bandwidth.

In conclusion, we make experiments on data set generated by simulation as well as dataset from real world, and the evaluation of these experiments further proves the superiority of DeepRS.

\section{CONCLUSION}

In this paper, we propose DeepRS, a deep learning based FEC system for real-time video streaming which mainly consists of two parts, LSTM model and RS encoder. DeepRS embeds an LSTM model inside and solves the problem of predicting the number of lost packets in the near future according to the historical packet loss pattern. With the help of LSTM model, DeepRS is able to automatically reduce redundancy ratio so as to prevent bandwidth waste under low packet loss rate, and increase redundancy ratio when packet loss is happening frequently. As for implementation, we have modified the LSTM model, thus adapting it to real scenarios in application. According to the results of experiments and evaluation, DeepRS achieves far better trade off between redundancy ratio and recovery ratio than traditional fix-redundancy FEC schemes. 


\section{REFERENCES}

[1] Cisco, "Cisco Visual Networking Index: Forecast and Trends, 20172022 White Paper", 2019, https://www.cisco.com/c/en/us/ \solutions/collateral/service-provider/visual-networking-index-vni/ white-paper-c11-741490.html

[2] Y. Wang and Q.-F. Zhu, "Error control and concealment for video communication: A review," Proceedings of the IEEE, vol. 86, no. 5, pp. 974-997, 1998.

[3] D. J. MacKay, "Fountain codes," IEE Proceedings-Communications, vol. 152, no. 6, pp. 1062-1068, 2005.

[4] U. Demir and O. Aktas, "Raptor versus reed solomon forward error correction codes," in International Symposium on Computer Networks, 2006.

[5] C. Bouras, N. Kanakis, V. Kokkinos, and A. Papazois, "Evaluating raptorq fec over 3 gpp multicast services," in Wireless Communications \& Mobile Computing Conference, 2012.

[6] S. B. Wicker and V. K. Bhargava, Reed-Solomon codes and their applications. John Wiley \& Sons, 1999.

[7] M. Sudan, "Decoding of reed solomon codes beyond the error-correction bound," Journal of complexity, vol. 13, no. 1, pp. 180-193, 1997.

[8] X. Yang, C. Zhu, G. L. Zheng, L. Xiao, and N. Ling, "An unequal packet loss resilience scheme for video over the internet," IEEE Transactions on Multimedia, vol. 7, no. 4, pp. 753-765, 2005.

[9] C. Padhye, K. J. Christensen, and W. Moreno, "A new adaptive fec loss control algorithm for voice over ip applications," in Performance, Computing, \& Communications Conference, Ipccc 00 Conference Proceeding of the IEEE International, 2000.

[10] A. A. E, S. G. Yoo, K. T. Chong, and H. Kim, "Packet loss rate prediction using the sparse basis prediction model," IEEE Transactions on Neural Networks, vol. 18, no. 3, pp. 950-954, 2007.

[11] S. L. Fong, S. Emara, B. Li, A. Khisti, W.-T. Tan, X. Zhu, and J. Apostolopoulos, "Low-latency network-adaptive error control for interactive streaming," arXiv preprint arXiv:1909.06709, 2019.

[12] S. Hochreiter and J. Schmidhuber, "Long short-term memory," Neural Computation, vol. 9, no. 8, pp. 1735-1780, 1997.

[13] A. Ganjam, F. Siddiqui, J. Zhan, X. Liu, I. Stoica, J. Jiang, V. Sekar, and H. Zhang, "C3: Internet-scale control plane for video quality optimization," in 12th \{USENIX\} Symposium on Networked Systems Design and Implementation ( $\{N S D I\}$ 15), 2015, pp. 131-144.

[14] J. Jiang, V. Sekar, I. Stoica, and H. Zhang, "Shedding light on the structure of internet video quality problems in the wild," in Proceedings of the ninth ACM conference on Emerging networking experiments and technologies. ACM, 2013, pp. 357-368.

[15] S. Fu, Z. Yan, Y. Jiang, C. Hu, and P. J. Marrn, "Experimental study for multi-layer parameter configuration of wsn links," in IEEE International Conference on Distributed Computing Systems, 2015. 